International Journal of Applied Mathematics

Volume 30 No. $4 \quad 2017,289-302$

ISSN: 1311-1728 (printed version); ISSN: 1314-8060 (on-line version)

doi: http://dx.doi.org/10.12732/ijam.v30i4.2

\title{
STUDY OF MHD FLOW OF BLOOD WITH HEAT TRANSFER IN AN ARTERIAL SEGMENT UNDER THE EFFECT OF PERIODIC BODY ACCELERATION
}

\author{
Mamata Parida \\ Department of Mathematics \\ Utkal University \\ Bhubaneswar, 751 004, INDIA
}

\begin{abstract}
The aim of this paper is to study numerically the blood flow in an arterial segment in the presence of an externally applied magnetic field and body acceleration by considering the fluid to be incompressible and Newtonian. A heat transfer analysis is carried out along with the governing momentum equations for the fully developed flow. A suitable numerical technique is employed to solve the Navier-Stokes equations. The influence of the applied magnetic field on the flow is analyzed with the aid of the dimensionless magnetic parameter $H$, the Hartmann number. The velocity distribution, temperature distribution and the heat transfer effects are studied and presented graphically.
\end{abstract}

AMS Subject Classification: 76Z05, 76W05, 80A20

Key Words: body acceleration, heat transfer, Hartmann number, finite volume method

Received: April 27, 2017

(c) 2017 Academic Publications 


\section{Introduction}

\section{List of symbols}

$\begin{array}{ll}a & \text { Dimensionless amplitude of body acceleration } \\ b & \text { Dimensionless frequency of body acceleration } \\ B_{o} & \text { Applied magnetic field strength } \\ C_{p} & \text { Specific heat at constant pressure } \\ E c & \text { Eckert number } \\ G(t) & \text { Dimensionless periodic body acceleration } \\ H & \text { Hartmann number } \\ p & \text { Dimensionless pressure } \\ P r & \text { Prandtl number } \\ R_{o} & \text { Half width of artery } \\ T_{w} & \text { Temperature of arterial wall } \\ u & \text { Dimensionless x-component of velocity } \\ v & \text { Dimensionless y-component of velocity } \\ \text { Greek } & \\ \alpha & \text { Womersley number } \\ \kappa_{o} & \text { Thermal conductivity } \\ \mu & \text { Viscosity of blood } \\ \omega & \text { Frequency of pulse } \\ \omega_{b} & \text { Frequency of body acceleration } \\ \phi_{g} & \text { Phase difference in body acceleration } \\ \rho & \text { Density of blood } \\ \sigma & \text { Electrical conductivity } \\ \theta & \text { Dimensionless temperature }\end{array}$

\section{Introduction}

The flow of blood through arteries is an important phenomenon in biofluid dynamics. In recent years, the study of magnetohydrodynamic (MHD) flow of blood has gained the attention of many researchers because of its wide range of physiological applications. Blood can be treated as a magnetic fluid because the erythrocytes contain haemoglobin molecules, which are oxides of iron and are present with uniquely high concentration in mature erythrocytes. Arteries are blood vessels that carry highly oxygenated blood away from the heart. They face high levels of blood pressure as they carry blood being pushed from the heart under great force. To withstand this pressure, the walls of the arteries are thicker, more elastic, and more muscular than those of other vessels. The 
smooth muscles of the arterial walls contract or expand to regulate the flow of blood through their lumen and hence control the amount of blood flow to different parts of the body under varying circumstances. Several features of biological flows such as vessel wall elasticity, non-Newtonian viscosity, slurry particles in the blood, although, are physiologically relevant but can be justifiably neglected in most of the arterial flows [1].

Vardanyan [2] studied that for steady flow in an artery, a uniform transverse magnetic field alters the flow rate of blood. It was observed by some investigators $[3,4,5]$ that under certain conditions blood exhibit visco-elastic behaviour which may be due to the visco-elastic properties of the individual red cells and the internal structures formed by cellular interactions. Pedley [6] and Fung et. al. [7] have conducted a study on the blood flow mechanics in arteries of different sizes. An experimental and numerical approach was adopted by Taylor and Draney [8] for quantifying the blood flow velocity and pressure field in human artery. A theoretical and experimental work was carrrried out by Berger et. al. [9] to provide an idea of the pressure drop and heat exchange in the fluid when it is subjected to move along a curved path. The effects of the interaction between a magnetic field and the haemodynamics of the arterial system have been studied in [10]. Misra and Chakravarty [11] have studied the unsteady flow of blood through arteries in which the blood was treated as a Newtonian viscous incompressible fluid. Several attempts have been made $[12,13,14]$ to study the effect of magnetic field on the blood flow in arteries in various physiological conditions.

In the present paper, the characteristics of blood flow and heat transfer is studied through parallel plates under the influence of periodic body acceleration in presence of transverse magnetic field. The study concentrates on the blood velocity, temperature, pressure and heat transfer rate with the variation of the magnetic field parameter $(H)$, body acceleration parameter $(\alpha)$ and thermal diffusivity parameter $(P r)$. The result of computation thus obtained for the physical quantities velocity, temperature and heat transfer are presented graphically. This investigation has clinical significance as it reveals the fact that the flow and the heat transfer rate of blood can be controlled by the application of sufficiently strong magnetic field.

\section{Governing equations}

Let us consider the unsteady two-dimensional laminar and fully developed flow of an incompressible and electrically conducting Newtonian fluid in a parallel 
plate channel bounded by the planes $y= \pm R_{0}$ (Fig. 1). A uniform magnetic field of strength $B_{0}$ is applied along the normal to the channel walls and the electrical conductivity $\sigma$ is assumed to be constant. Let $\left(x^{\star}, y^{\star}\right)$ be the material point in Cartesian coordinate system, $x^{\star}$ being the axis along the flow direction and $y^{\star}$ being perpendicular to it. The flow is considered to be symmetric about the $x$ - axis and our focus is on the flow profile in the region $0 \leq y \leq R_{0} . u^{\star}$, $v^{\star}$ are the velocity components in the $x^{\star}$ and $y^{\star}$ directions, respectively, and $t^{\star}$ is the time parameter. For $t^{\star}>0$, the flow is assumed to have periodic body acceleration given by

$$
G^{\star}\left(t^{\star}\right)=a^{\star} \cos \left(\omega_{b} t^{\star}+\phi_{g}\right),
$$

where $a^{\star}$ is the amplitude of the body acceleration.

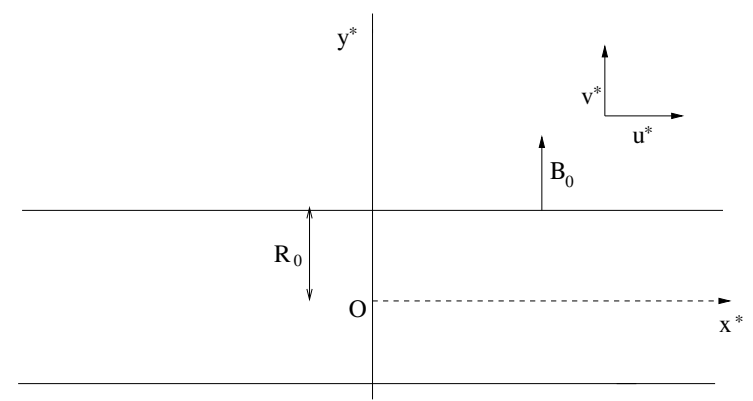

Figure 1: Schematic of the flow configuration of the artery.

The governing equations of the unsteady two-dimensional flow of the viscous incompressible fluid are given by

$$
\begin{aligned}
\frac{\partial u^{\star}}{\partial x^{\star}}+\frac{\partial v^{\star}}{\partial y^{\star}}= & 0, \\
\rho\left(\frac{\partial u^{\star}}{\partial t^{\star}}+u^{\star} \frac{\partial u^{\star}}{\partial x^{\star}}+v^{\star} \frac{\partial u^{\star}}{\partial y^{\star}}\right)= & -\frac{\partial p^{\star}}{\partial x^{\star}}-\sigma B_{0}^{2} u^{\star}+\rho G^{\star}\left(t^{\star}\right) \\
& +\mu\left(\frac{\partial^{2} u^{\star}}{\partial x^{\star 2}}+\frac{\partial^{2} u^{\star}}{\partial y^{\star 2}}\right) \\
\rho\left(\frac{\partial v^{\star}}{\partial t^{\star}}+u^{\star} \frac{\partial v^{\star}}{\partial x^{\star}}+v^{\star} \frac{\partial v^{\star}}{\partial y^{\star}}\right)= & -\frac{\partial p^{\star}}{\partial y^{\star}} \\
& +\mu\left(\frac{\partial^{2} v^{\star}}{\partial x^{\star 2}}+\frac{\partial^{2} v^{\star}}{\partial y^{\star 2}}\right) \\
\rho C_{p}\left(\frac{\partial T^{\star}}{\partial t^{\star}}+u^{\star} \frac{\partial T^{\star}}{\partial x^{\star}}+v^{\star} \frac{\partial T^{\star}}{\partial y^{\star}}\right)= & \kappa_{0}\left(\frac{\partial^{2} T^{\star}}{\partial x^{\star 2}}+\frac{\partial^{2} T^{\star}}{\partial y^{\star 2}}\right)
\end{aligned}
$$




$$
+\sigma B_{0}^{2} u^{\star 2}
$$

along with the following boundary conditions on velocity and temperature

$$
\begin{aligned}
& \frac{\partial u^{\star}}{\partial y^{\star}}=0, \quad \frac{\partial T^{\star}}{\partial y^{\star}}=0 \quad \text { at } \quad y^{\star}=0, \\
& u^{\star}=v^{\star}=0, \quad T^{\star}=T_{w} \quad \text { at } \quad y^{\star}=R_{0} .
\end{aligned}
$$

We now introduce the non-dimensional variables defined by

$$
\begin{aligned}
& x=\frac{x^{\star}}{R_{0}}, \quad y=\frac{y^{\star}}{R_{0}}, \quad u=\frac{u^{\star}}{\omega R_{0}}, \quad \nu=\frac{\nu^{\star}}{\omega R_{0}}, \\
& p=\frac{p^{\star}}{\mu \omega}, \quad t=t^{\star} \omega, \quad \theta=\frac{T^{\star}}{T_{w}} .
\end{aligned}
$$

Using these dimensionless variables, equations (2)-(5) become

$$
\begin{aligned}
\frac{\partial u}{\partial x}+\frac{\partial v}{\partial y}= & 0 \\
\frac{\partial u}{\partial t}+u \frac{\partial u}{\partial x}+v \frac{\partial u}{\partial y}= & -\frac{1}{\alpha^{2}} \frac{\partial p}{\partial x}+\frac{1}{\alpha^{2}}\left(\frac{\partial^{2} u}{\partial x^{2}}+\frac{\partial^{2} u}{\partial y^{2}}\right) \\
& -\frac{H^{2}}{\alpha^{2}} u+\frac{1}{\alpha^{2}} G(t), \\
\frac{\partial v}{\partial t}+u \frac{\partial v}{\partial x}+v \frac{\partial v}{\partial y}= & -\frac{1}{\alpha^{2}} \frac{\partial p}{\partial y}+\frac{1}{\alpha^{2}}\left(\frac{\partial^{2} v}{\partial x^{2}}+\frac{\partial^{2} v}{\partial y^{2}}\right) \\
\frac{\partial \theta}{\partial t}+u \frac{\partial \theta}{\partial x}+v \frac{\partial \theta}{\partial y}= & \frac{1}{\alpha^{2} P r}\left(\frac{\partial^{2} \theta}{\partial x^{2}}+\frac{\partial^{2} \theta}{\partial y^{2}}\right)+\frac{H^{2} E c}{\alpha^{2}} u^{2}
\end{aligned}
$$

where $\alpha=R_{0} \sqrt{\frac{\omega \rho}{\mu}}$ is the Womersley number, $H=R_{0} B_{0} \sqrt{\frac{\sigma}{\mu}}$ is the Hartmann number, $\operatorname{Pr}=\frac{\mu C_{p}}{\kappa_{0}}$ is the Prandtl number, $E c=\frac{\omega^{2} R_{0}^{2}}{C_{p} T_{w}}$ is the Eckert number, $G(t)=a \cos \left(b t+\phi_{g}\right), a=\frac{\rho R_{0}}{\omega \mu} a^{\star}$ and $b=\frac{\omega_{b}}{\omega}$.

The corresponding boundary conditions are

$$
\begin{aligned}
& \frac{\partial u}{\partial y}=0, \quad \frac{\partial \theta}{\partial y}=0 \quad \text { at } \quad y=0, \\
& u=v=0, \quad \theta=1 \quad \text { at } \quad y=1 \text {. }
\end{aligned}
$$

The Nusselt number is obtained by calculating the temperature gradient on the plates from the relation

$$
N u=\left.\frac{x}{T_{w}} \frac{\partial \theta}{\partial y}\right|_{y=0} .
$$




\section{Numerical methods}

In order to discretize the non-linear partial differential equations we have opted the finite volume method. The problem domain $\Omega$ has been decomposed into a finite number of discrete network of non-overlapping cells. The conservative form of the governing equations is

$$
\frac{\partial \vec{U}}{\partial t}+\frac{\partial \vec{F}_{i}}{\partial x_{i}}+\frac{\partial \vec{G}_{i}}{\partial x_{i}}=\vec{B}
$$

where $\vec{U}, \vec{F}_{i}, \vec{G}_{i}$ and $\vec{B}$ are the conservation flow variables, convection flux variables, diffusion flux variables and source terms, respectively. Integrating Eq. (14) spatially over the control volume $\Omega$ and applying Gauss integral theorem, we obtain

$$
\int_{\Omega}\left(\frac{\partial \vec{U}}{\partial t}-\vec{B}\right) d \Omega+\int_{\Gamma}\left(\vec{F}_{i}+\vec{G}_{i}\right) n_{i} d \Gamma=0,
$$

where $\Gamma$ is the surface of the control volume and $n_{i}$ are the components of the unit normal vector to the surface. We have considered rectangular control volumes with cell centered arrangement of nodes given in Fig. 2.

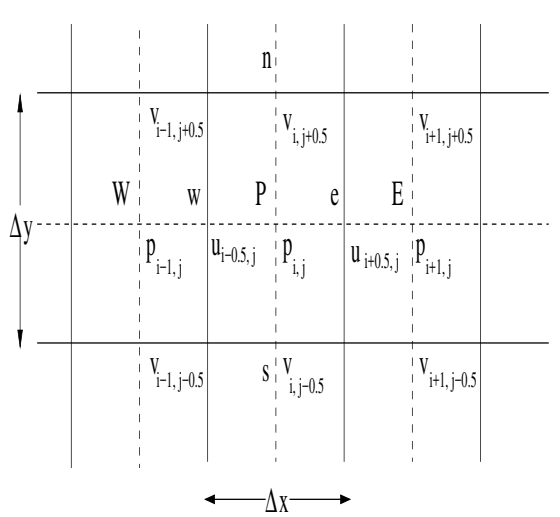

Figure 2: Cell-centered arrangement of nodes.

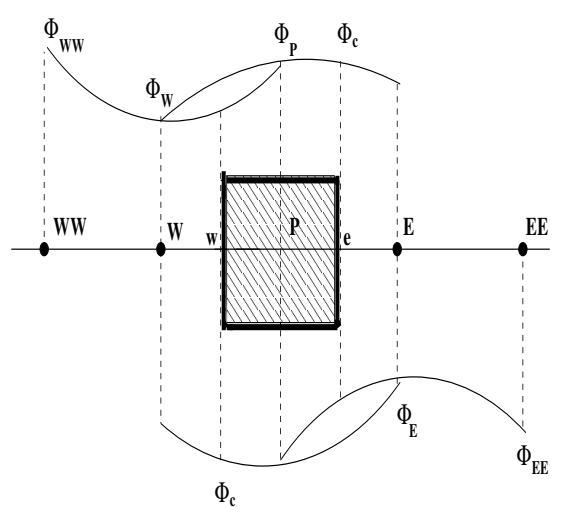

Figure 3: Quadratic upstream based interpolation for $\mathrm{u}[15$, $16]$.

The choice of averaging fluxes has been employed for the approximation of the convective and diffusive fluxes through the control volume faces. As a result of the splitting of the surface integral in Eq. (15) into the sum of four surface 
integrals over the cell faces $\Gamma_{c}(c=e, w, n, s)$ of the control volume, Eq. (15) becomes

$$
\begin{gathered}
\int_{\Omega}\left(\frac{\partial \vec{U}}{\partial t}-\vec{B}\right) d \Omega+\sum_{c} \int_{\Gamma_{c}}\left(\vec{F}_{c i}+\vec{G}_{c i}\right) n_{c i} d \Gamma_{c}=0, \\
\phi_{c}=\left\{\begin{array}{lll}
a_{1} \phi_{E}-a_{2} \phi_{W}+\left(1-a_{1}+a_{2}\right) \phi_{P} & \text { if } & A_{\phi}>0 \\
b_{1} \phi_{P}-b_{2} \phi_{E E}+\left(1-b_{1}+b_{2}\right) \phi_{E} & \text { if } & A_{\phi}<0
\end{array},\right.
\end{gathered}
$$

where $A_{\phi}$ is the coefficient of $\phi_{c}$ through the face $\Gamma_{c}$. As we have considered an equidistant grid

$$
\begin{aligned}
& a_{1}=b_{1}=\frac{3}{8}, \\
& a_{2}=b_{2}=\frac{1}{8} .
\end{aligned}
$$

The diffusive terms are discretized by the finite difference method using the central difference scheme. Integrating the continuity equation over the $(i, j)^{t h}$ scalar control volume at the $(n+1)^{t h}$ time level gives

$$
\left(u_{i, j}^{n+1}-u_{i-1, j}^{n+1}\right) \Delta x+\left(v_{i, j}^{n+1}-v_{i, j-1}^{n+1}\right) \Delta y=0 .
$$

Integrating the $x$-momentum equation at the $(n+1)^{t h}$ time step over $(i, j)^{t h}$ control volume for $u$ produces

$$
\begin{aligned}
& \left(\frac{\Delta x \Delta y}{\Delta t}+a_{i, j}^{u}\right) u_{i, j}^{n+1}+\sum a_{n b}^{u} u_{n b}^{n+1}=\left(\frac{\Delta x \Delta y}{\Delta t}-\frac{H^{2}}{\alpha^{2}}\right) u_{i, j}^{n} \\
& -\frac{1}{\alpha^{2}}\left\{\Delta y\left(p_{i+1, j}^{n+1}-p_{i, j}^{n+1}\right)-G(t)\right\},
\end{aligned}
$$

where

$$
\begin{aligned}
a_{i, j}^{u}= & \left(\frac{2}{\alpha^{2}}\right)\left(\frac{\Delta y}{\Delta x}+\frac{\Delta x}{\Delta y}\right)+\frac{1}{4}\left(u_{i+1, j}^{n}-u_{i-1, j}^{n}\right) \Delta y \\
& +\frac{1}{4}\left(v_{i, j}^{n}+v_{i+1, j}^{n}-v_{i, j-1}^{n}-v_{i+1, j-1}^{n}\right) \Delta x .
\end{aligned}
$$

$\sum a_{n b}^{u} u_{n b}^{n+1}$ denotes all the convection and diffusion contribution from neighbouring nodes $(i-1, j),(i+1, j),(i, j-1)$ and $(i, j+1)$. The term $\Delta y\left(p_{i+1, j}^{n+1}-\right.$ 
$\left.p_{i, j}^{n+1}\right)$ represents the pressure acting on the $u$-control volume. Similarly, the $y$-momentum and energy equations can be discretized as

$$
\begin{aligned}
& \left(\frac{\Delta x \Delta y}{\Delta t}+a_{i, j}^{v}\right) v_{i, j}^{n+1}+\sum a_{n b}^{v} v_{n b}^{n+1}=\frac{\Delta x \Delta y}{\Delta t} v_{i, j}^{n}-\frac{1}{\alpha^{2}} \\
& \times\left\{\Delta x\left(p_{i, j+1}^{n+1}-p_{i, j}^{n+1}\right)\right\}, \\
& \left(\frac{\Delta x \Delta y}{\Delta t}+a_{i, j}^{\theta}\right) \theta_{i, j}^{n+1}+\sum a_{n b}^{\theta} \theta_{n b}^{n+1}=\frac{\Delta x \Delta y}{\Delta t} \theta_{i, j}^{n}+\frac{H^{2} E c}{\alpha^{2}} \\
& \times\left(u_{i, j}^{n+1} u_{i, j}^{n} \Delta x \Delta y\right),
\end{aligned}
$$

where

$$
\begin{aligned}
a_{i, j}^{v}= & \left(\frac{2}{\alpha^{2}}\right)\left(\frac{\Delta y}{\Delta x}+\frac{\Delta x}{\Delta y}\right)+\frac{1}{4}\left(v_{i, j+1}^{n}-v_{i, j-1}^{n}\right) \Delta x \\
& +\frac{1}{4}\left(u_{i, j}^{n}+u_{i, j+1}^{n}-v_{i-1, j+1}^{n}-v_{i-1, j}^{n}\right) \Delta y, \\
a_{i, j}^{\theta}= & \left(\frac{2}{\alpha^{2} P r}\right)\left(\frac{\Delta y}{\Delta x}+\frac{\Delta x}{\Delta y}\right)+\frac{1}{4}\left(\theta_{i+1, j}^{n}-\theta_{i-1, j}^{n}\right) \Delta y \\
+ & \frac{1}{4}\left(\theta_{i, j+1}^{n}-\theta_{i, j-1}^{n}\right) \Delta x .
\end{aligned}
$$

A convenient combination of the tridiagonal matrix algorithm (TDMA) method and the Gauss-Seidel method has been implemented to solve the resulting system of algebraic equations. We have made use of the semi-implicit method for pressure linked equations (SIMPLE) algorithm for pressure correction. This algorithm is based on a cyclic series of guess-and-correct operations and represents an implicit influence of the pressure correction on velocity. All velocity components are first calculated from the momentum equations using a guessed pressure field. Then the pressure and velocities are corrected so as to satisfy continuity. This process continues until the solution converges. The pressure link between the continuity and momentum equations is accomplished by transforming the continuity equation into a Poisson equation for pressure. The Poisson equation implements a pressure correction for a divergent velocity field. The solution of the stated unsteady problem involves the process of solving a succession of steady state problems. For reasonable values of time step $\Delta t$, the known $\phi$ values at time $t$ are used as a guess of the unknown $\phi$ values at time $t+\Delta t$, 
where $\phi$ denotes the dependent variables of the problem. Iteration at each time step continues until the divergence-free solution is obtained. We have obtained a time-independent numerical solution which is convergent by advancing the flow field variables through a sequence of shorter time step $\Delta t=0.001$. For the range of parameter values considered here, the flow field achieves a steady state after a transient state, and this steady state is independent of the initial conditions prescribed. For achieving a satisfactory convergence of this scheme we have used the under-relaxation parameter $\beta=0.8$ in $p=\bar{p}+\beta p^{\prime}$, where $\bar{p}$ is the guessed pressure, $p^{\prime}$ is the pressure correction and $p$ is the actual pressure. The convergence criteria is employed of the form

$$
\left|\phi_{i, j}{ }^{n+1}-\phi_{i, j}{ }^{n}\right|<\varepsilon .
$$

Here $i$ and $j$ denote the cell indices, $n$ is the time level, $\phi$ stands for $u, v$ or $\theta$ and the value of $\varepsilon$ is considered to be $10^{-4}$.

\section{Results and discussion}

The blood flow phenomenon and heat transfer through a normal artery in the presence of periodic body acceleration as well as an external magnetic field is studied. The flow is governed by the Womersley number, the Hartmann number and the Prandtl number. The physiological applicable data used for computation of numerical results are collected from the existing literatures and are listed as: $\alpha=3.0 ; H=1.0 ; \operatorname{Pr}=21.0 ; b=1.0 ; a=1.0 ; \phi_{g}=0.0 ; R_{0}$ $=1.0 \mathrm{~mm} ; E c=0.0002 ; T_{w}=310 \mathrm{~K} ; \rho=1,050 \mathrm{~kg} / \mathrm{m}^{3} ; B_{0}=8$ Tesla and $\sigma$ $=0.8 \mathrm{~s} / \mathrm{m}$. The computational results are presented graphically for different values of the parameters involved in the present problem. In all the numerical computations that has been carried out and presented in form of figures we have adopted the steady state condition considering time $t=0.5$.

Fig. 4 depicts the variation of axial velocity for different values of Hartmann number with Womersley number $\alpha=3$ and Prandtl number $\operatorname{Pr}=21$. It reveals that the velocity decreases as the the magnetic strength parameter increases. In arteries the magnetic field strength brings a great change in the $u$-velocity of blood. When the biomagnetic fluid (blood) is subjected to a magnetic field, the action of magnetization introduces an orientation of the blood charged ions with the magnetic field. The action of orientation makes the red blood cells more suspended in the plasma and an increase in the haemoglobin concentration results in an increase in the internal blood viscosity, and hence a reduction in blood axial flow velocity is obtained. This result supports the phenomenon that 


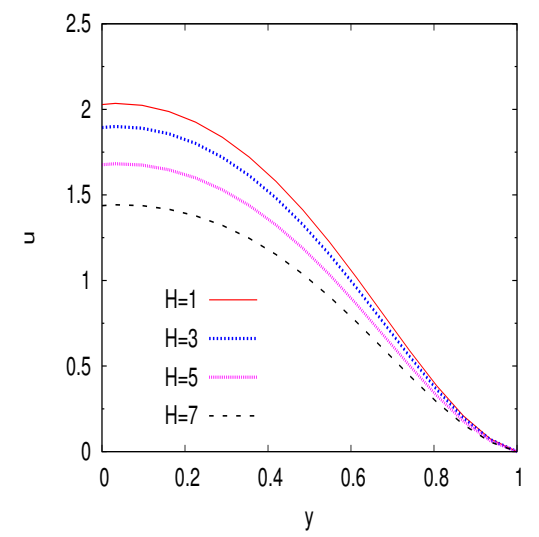

Figure 4: Variation of axial velocity profile for different values of $H$.

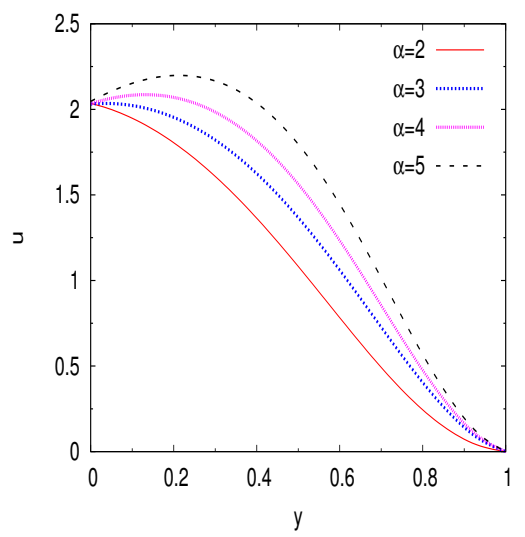

Figure 5: Variation of axial velocity profile for different values of $\alpha$.

decreasing magnetic strength reduces the flow resistance and hence the velocity of blood flow increases with the decrease in magnetic strength. The maximum velocity of blood occurs in the middle of the channel and gradually reduces to zero on the walls as we have considered the arterial wall to be a rigid plate and hence no slip condition prevails.

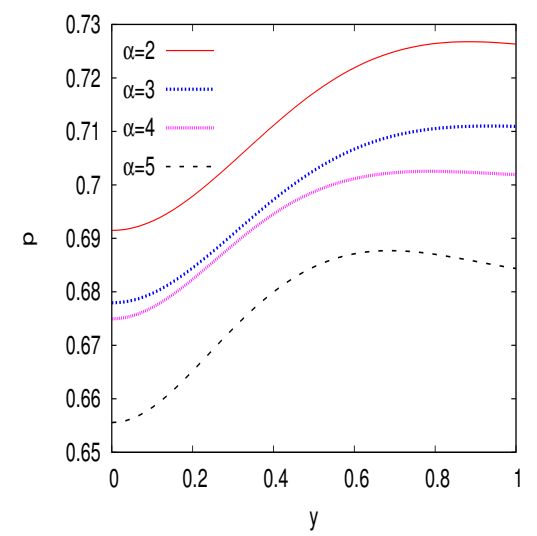

Figure 6: Distribution of dimensionless pressure $\mathrm{p}$ for different values of $\alpha$.

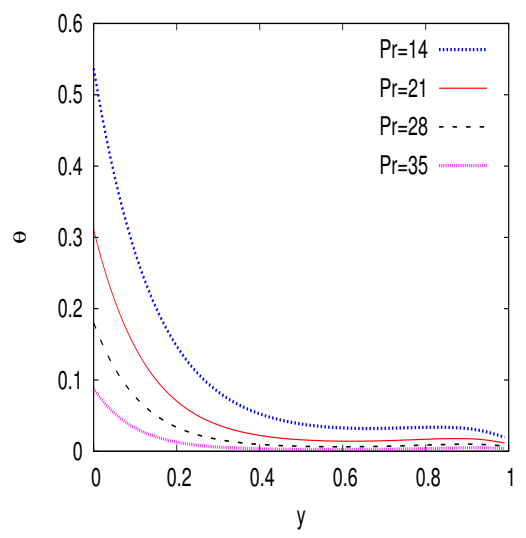

Figure 7: Distribution of dimensionless temperature $\theta$ for different values of $\mathrm{Pr}$.

The axial velocity profile for various values of Womersley number is included 
in Fig. 5. It is observed that the axial velocity increases with increasing body acceleration parameter, the Womersley number. This is due to the fact that whenever the body accelerates faster the requirement of blood in the heart increases and for compensating this, blood flow rate increases in the arteries. The non-dimensional pressure distribution with varying $\alpha$ is presented in Fig. 6 .

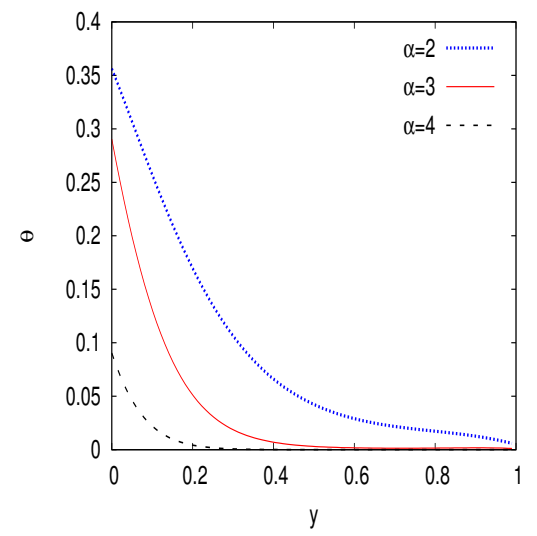

Figure 8: Distribution of dimensionless temperature $\theta$ for different values of $\alpha$.

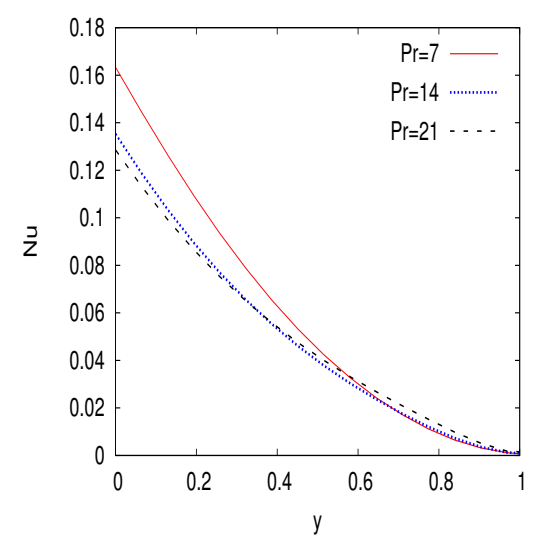

Figure 9: Heat transfer rate for different values of $\mathrm{Pr}$.

The non-dimensional pressure decreases with increasing value of the body acceleration parameter. The pressure $p$ attains its minimum in the middle of the channel, then goes on increasing and takes almost a constant value when approaches the arterial wall. This suggests that in blood flow through an artery the maximum pressure is exerted on the walls. The distribution of non-dimensional temperature is shown in figures 7 and 8 for different Prandtl numbers and Womersley numbers, respectively. It is interesting to find that temperature decreases with increasing thermal diffusivity parameter $(P r)$ and the body acceleration parameter. It is often found that after running or jogging, where the body gets accelerated there is sweating which gives a cooling effect that is the temperature inside our body gets lowered down. The maximum heat transfer occurs in the middle of the channel as can be seen in figures 9- 11 and reduces to zero on the walls. In case of varying Prandtl number (Fig. 9), the variation found in the heat transfer rate from $\operatorname{Pr}=7$ to 14 is very much prominent as compared to the variation occuring from $\operatorname{Pr}=14$ to 21 . Fig. 10 infers that the heat transfer rate decreases by increasing the magnetic parameter $H$. Thus, by applying a magnetic field of sufficient strength the heat 
transfer rate can be controlled which has clinical significance during surgeries and cancer therapy. For varying Womersley number, the heat transfer rate decreases with increasing $\alpha$ in the beginning and then increases with increasing $\alpha$ when approaches the wall as shown in Fig. 11.

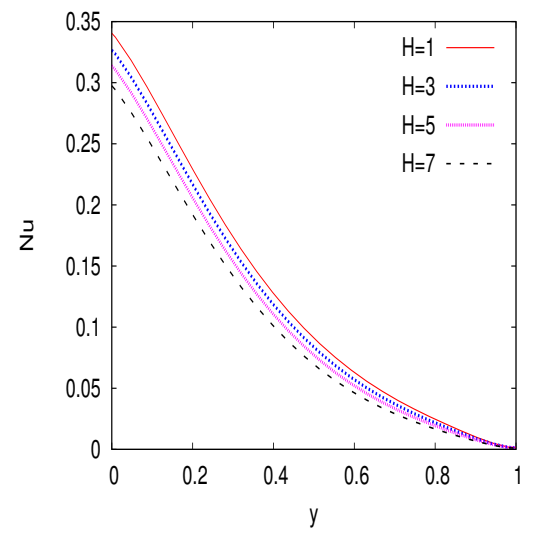

Figure 10: Heat transfer rate for different values of $H$.

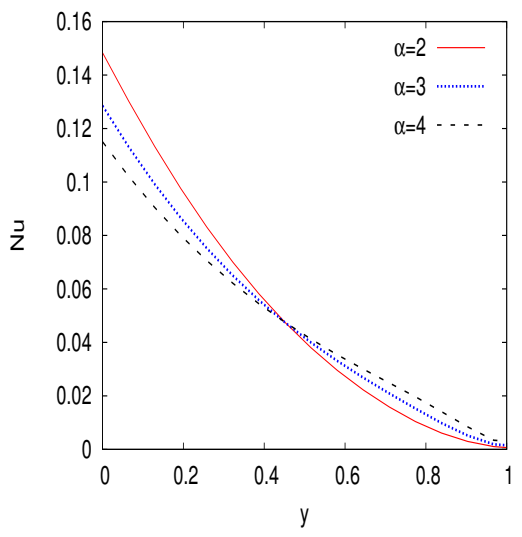

Figure 11: Heat transfer rate for different values of $\alpha$.

\section{Conclusion}

The present study is motivated towards the flow and heat transfer of blood in an artery under the combined effect of periodic acceleration and an external magnetic field. Blood behaves as a non-Newtonian fluid in channels of narrow width which are referred as arterioles and capillaries whereas its behavior is Newtonian in most of the arteries. We have considered such an artery as our channel. We have made an attempt to examine the effect of the Prandtl number, the Hartmann number and the Womersley number on the flow and heat transport characteristic of blood. Some graphical presentations of the computed results have been performed. The study reveals that the axial velocity and the heat transfer rate can be controlled by the application of a strong magnetic field. The observations also indicate the influence of the body acceleration parameter $\alpha$ on the axial velocity, pressure and temperature distribution. Furthermore, increasing Prandtl number results in decreasing temperature as well as heat transfer rate. This investigation has significant clinical importance during the stages where the blood flow need to be checked (surgery) and the heat transfer rate to be controlled (therapy). 


\section{References}

[1] N.Ku. David, Blood flow in arteries, Ann. Rev. of Fluid Mechanics, 29 (1997), 399-434.

[2] V.A. Vardanyan, Effect of magnetic field on blood flow, Biofizika, 18 (1973), 491-496.

[3] G.B. Thurston, Viscoelasticity of human blood, Biophys J., 12 (1972), 1205-1217.

[4] J.F. Stoltz, M. Lucius, Viscoelasticity and thixotropy of human blood, Biorheology, 18 (1981), 453-473.

[5] E. Fukada, M. Kaibara, Viscoelastic study of aggregation of red blood cells, Biorheology, 17 (1980), 177-182.

[6] T.J. Pedley, The fluid mechanics of large blood vessels, Cambridge University Press (1980).

[7] Y.C. Fung, B.W. Zweifach, Microcirculation, mechanics of blood flow in capillaries, Ann. Rev. of Fluid Mechanics, 3 (1971), 189-210.

[8] C.A. Taylor, M.T. Draney, Experimental and computational methods in cardiovascular fluid mechanics, Ann. Rev. of Fluid Mechanics, 36 (2004), 197-231.

[9] S.A. Berger, L. Talbot, L.S. Yao, Flow in curved pipes, Ann. Rev. of Fluid Mechanics, 15 (1983), 461-512.

[10] V.K. Sud, G.S. Sekhon, Blood flow through the human arterial system in the presence of a steady magnetic field, Phys. Med. Biol., 34 (1989), 795-805.

[11] J.C. Misra, S. Chakravarty, Flow in arteries in the presence of stenosis, J. Biomech., 19 (1986), 907-918.

[12] E.E. Tzirtzilakis, A mathematical model for blood flow in magnetic field, Phys. Fluids, 17 (2005), ID 077103.

[13] J.C. Misra, G.C. Shit, Effect of magnetic field on blood flow through an artery: A numerical model, Computational Technologies, 12 (2007), 3-16. 
[14] A. Ogulu, E. Amos, Modeling pulsatile blood flow within a homogeneous porous bed in the presence of a uniform magnetic field and time-dependent suction, Int. Commun. Heat and Mass Transf., 34 (2007), 989-995.

[15] W. Shyy, S. Thakur, J. Wright, Second-Order Upwind and Central Difference Schemes for Recirculating Computation, AIAA Journal, 30 (1992), 923-932.

[16] B.P. Leonard, A stable and accurate convective modeling procedure based on quadratic upstream interpolation, Comput. Meth. Appl. Mech. Eng., 19 (1979), 59-98. 